\title{
Stiffness Discrimination by Two Fingers with Stochastic Resonance
}

\author{
Komi Chamnongthai $\left.{ }^{(}\right)$, Takahiro Endo, Shohei Ikemura, \\ and Fumitoshi Matsuno \\ Kyoto University, Kyoto 615-8540, Japan \\ chamnongthai.komi.46m@st.kyoto-u.ac.jp
}

\begin{abstract}
This paper focuses on Stochastic Resonance (SR) for stiffness discrimination by two fingers. In particular, we show that the subthreshold vibrotactile noise applied on a remote position can improve tactile sensations of both index finger and thumb for a task requiring multiple fingers. We evaluate the user performances in a virtual environment (VE) by Weber fraction for stiffness perception under one of three different vibration source positions: on the index finger, on the thumb, and between the index finger and thumb. The results show that the stiffness discrimination ability increase under all three vibration source positions with the best performance obtained for the source location between index finger and thumb. The finding indicates the potential of using a single vibration source to enhance sensation of multiple fingers by the effect of SR.
\end{abstract}

Keywords: Stiffness discrimination - Stochastic Resonance $\cdot$ Haptic interface $\cdot$ Multiple fingers

\section{Introduction}

In clinical field, palpation is one process to identify properties such as size, texture, location, etc., of an organ by a physical examination with multiple fingers. The ability to address the abnormality of the organ stiffness is one vital skill before operating other processes. However, to achieve the expert level performance to distinguish the difference accurately, the medical students must be trained strenuously with various models and difficulties. Furthermore, training with traditional methods consume a lot of time to obtain new skills. In addition, it also costs a large amount of money to prepare the instruction equipment for covering the various difficulties of training. To solve this problem, a haptic training system which combines a virtual environment (VE) and haptic interface is one interesting solution.

The common approach followed to communicate with haptic training system is to design a finger holder into which the user will insert his or her finger. However, the use of a finger holder decreases the force-detection ability at the finger [1] and therefore it is necessary to enhance the force-detection capability of the finger through other mechanisms in the presence of finger holder.

(C) The Author(s) 2020

I. Nisky et al. (Eds.): EuroHaptics 2020, LNCS 12272, pp. 497-505, 2020.

https://doi.org/10.1007/978-3-030-58147-3_55 
There are several techniques to enhance tactile sensation: transcutaneous electrical nerve stimulation [2], temporary deafferentation [3], and passive sensory stimulation [4]. One effective solution is the addition of sensory noise which provides vibrotactile noise through the human skin in order to boost the sensitivity in several body parts such as the feet [5] and fingers [6]. This phenomena, which boosts the weak signal to be detectable by adding white noise, is called Stochastic Resonance (SR).

There are numerous studies that show the increase of haptic sensitivity by the effect of SR. According to Kurita et al. [8], the effect of SR is applied directly to the tip of the index finger, which shows the improvement of the user performance in grasping task. Furthermore, the effect of SR does not only occur when the vibration source is close to the tip of the index finger, but also happen while the vibration is generated from remote positions, which is away from the tip of the finger [9]. The study shows that the haptic sensation of the user is enhanced when a subthreshold vibration with a remote position is applied to the stroke patients. The fingertip perception is also shown to improve using SR when the finger is enclosed in the finger holder [10]. Therefore, the effect of SR has a potential to raise the haptic sensation at the user fingertip. However, there is no study that investigates the effects of SR on the fingertip when multiple fingers are enclosed within finger holders while doing the motor task.

The goal of this study is to determine the effect of SR in stiffness discrimination task using two fingers in VE. For this, we propose a novel method which consists of haptic feedback generated by haptic devices and the effect of SR provided by a piezoelectric actuator, in a stiffness discrimination task which is manipulated by multi-fingers through VE. The user performances are evaluated with three different vibration source positions (on the index finger (Position 1), between the index finger and thumb (Position 2), on the thumb (Position 3)) in order to find the possibility of the enhancement of the sensitivities at the fingertips. Many papers address that the haptic sensation of one finger is increased by one vibration source, but in this paper, we newly reveal that even one vibration source can improve the sensations of the two fingers via the effect of SR.

\section{Proposed Method}

We propose a method which integrates haptic feedback and the effect of SR for a better haptic sensitivity in the stiffness discrimination task. To provide the effect of SR, the mechanical vibration is applied to one of the three positions shown in Fig. 1 with varying vibration intensities. We would like to investigate the possibility that one vibration source can enhance the sensation of both fingers via the effect of SR. Two haptic devices, both being Geomagic Touch haptic devices, are used in this study. The original end-effectors of the haptic devices are customized for the task to operate with a finger as shown in Fig. 2(b). The modified end-effector consists of the finger holder, made from polyoxymethylene, and a force sensor (Leptrino, CFS018CA101U). On the other hand, the VE is programmed by using the CHAI3D library [11]. 


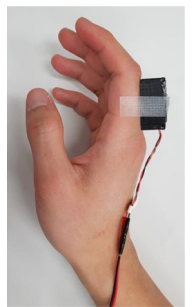

(a)

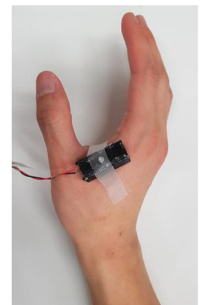

(b)

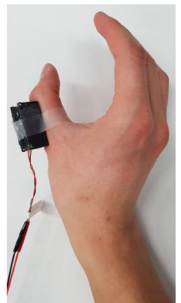

(c)

Fig. 1. The vibration source position in this study (a) on the index finger (Position 1), (b) between the index finger and the thumb (Position 2), (c) on the thumb (Position 3).

A piezoelectric actuator (Cedrat Technology Inc.: APA120S) is placed at the one of the three positions of the user hands to generate additive white Gaussian noise and gain the effect of SR. Additionally, the piezoelectric actuator can control the amplitude and the frequency of displacement freely, thus, the complicated signal is possible to generate. The generated vibration frequency is low-pass filtered at $400 \mathrm{~Hz}$ in order to activate all mechanoreceptors, as Pacinian corpuscles are active at frequencies between 0.5 and $400 \mathrm{~Hz}$ [12]. The Box-Muller method [13] is used to generate the white gaussian noise vibration $x(t)$ through the piezoelectric actuator:

$$
x(t)=\sigma \sqrt{-2 \ln \alpha(t)} \sin (2 \pi \beta(t)),
$$

where $t$ is time, $\sigma$ is the noise intensity, and $\alpha$ and $\beta$ are independent random variables in the interval $(0,1)$, and $x(t)$ corresponded to the voltage.

\section{Evaluation Method}

\subsection{Subjects}

Six healthy participants (mean age \pm SD: $24.5 \pm 1.88$ years, all male) participated in the study. Before doing the experiment, all participants understood and consented to the experimental protocol approved by the Institutional Review Board of the Graduate School of Engineering, Kyoto University (No. 201707). The Weber Fraction (WF) value of stiffness perception was used to compare the user performance to detect the fingertip force. The participants inserted their index finger and thumb in the finger holders which are attached to the haptic devices, while VE in the computer monitor displayed two virtual objects to the participants.

\subsection{Experimental Procedure}

In this experiment, the sensory threshold $(T)$ of each participant was measured using the the staircase-method [7] as the lowest vibration intensity that could be 
felt by the participants. Then participants performed the task with each of seven different vibration intensities; i.e., no vibration $(0 T), 40 \%(0.4 T), 50 \%(0.5 T)$, $60 \%(0.6 T), 70 \%(0.7 T), 80 \%(0.8 T)$, and $100 \%(1.0 T)$ of the sensory threshold with three different positions. The vibration intensities were provided randomly to participants to avoid learning effects. Furthermore, participants wore passive noise-cancelling headset to avoid hearing the vibration sound.

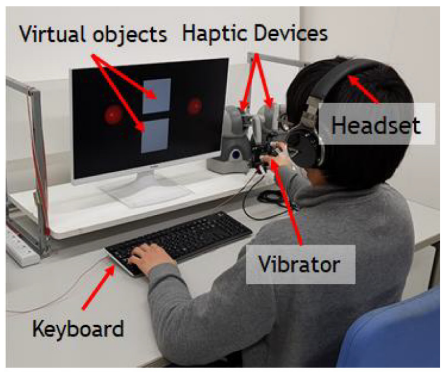

(a)

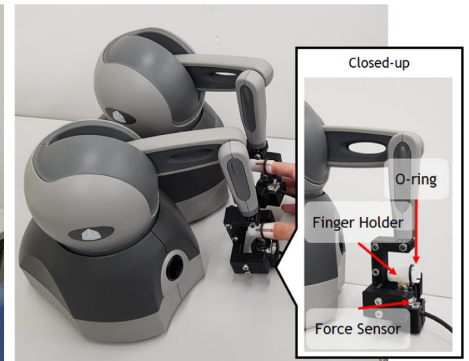

(b)

Fig. 2. Overview of experimental setup. (a) experimental setup when the participant was performing the task. The participants gets a force feedback through the haptic devices when he or she touches a virtual object through the red cursors which represent the finger positions on the screen, (b) the modified haptic devices were used in the study. The finger holder is attached to the device arm.

In VE, two virtual objects were presented as shown in Fig. 2(a). The objects were displayed as non-deformable in order to avoid the effect of visual feedback. The reference stiffness values were selected to be close to that of body fat of breast tissue $(35 \mathrm{~N} / \mathrm{m}$ [14]) as one of 25,35 and $55 \mathrm{~N} / \mathrm{m}$. One virtual object had one of four reference stiffness value whereas the other object started with corresponding difference of $10 \mathrm{~N} / \mathrm{m}$ and changed afterward as described later. The participants could touch the virtual objects for as long as they wanted. The force feedback was calculated using Hook's law; i.e., by multiplying the depth of the penetration of the finger into the virtual object and the stiffness of the touched object. The participants touched the two virtual objects and then chose the stiffer object by pressing a designated key on the keyboard, before starting the next trial. The task was completed by the white objects turning red. Average time spent on the experiment was around $2.5 \mathrm{~h}$ for one participant, including rest time.

For each reference value if the first object, Wald rule [15] was used to decide when to change the comparison stiffness for altering the stiffness of the second object. Furthermore, the change of the stiffness amount was decided by PEST rule [15], because the fixed step size takes longer time to complete the task, which lead the participant frustrated. A reversal point is defined as the turning point after which change in stiffness goes to the opposite direction of the previous direction. The average of the last four reversal values is used to determine the 
just noticeable difference (JND) of stiffness perception. Then we calculated WF of the subject in each condition as the average value between JND and reference stiffness:

$$
W F=\frac{J N D}{\text { Reference Stiffness }} .
$$

\section{Experimental Results and Discussion}

The average WF values of each vibration intensity and stiffness difference are shown in three sub-figures of Fig. 3. The vertical axes show the WF value while the horizontal axes show the reference stiffness in each session. For example, a $35 \mathrm{~N} / \mathrm{m}$ of reference stiffness with $0.6 T$ is a point at $35 \mathrm{~N} / \mathrm{m}$. A lower WF value implies a higher haptic sensitivity of the user.

The results show that the WF values in all vibration-existed conditions tend to be less than the WF in no-vibration condition. As shown in Fig. 3, there are significant differences $(p<0.05)$ in the average WF values at $0 T$ and $0.6 T$ in all three sub-figures, confirmed by a two-tailed paired $t$-test. Moreover, the average of performance at $0.6 T$ has the lowest $\mathrm{WF}$ values among other vibration levels.

Table 1. Results of Anova (Single Factor) in each vibration level

\begin{tabular}{|c|c|c|c|c|}
\hline \multicolumn{5}{|c|}{ ANOVA (Single Factor) } \\
\hline \multirow{2}{*}{ Vibration Intensity } & \multicolumn{5}{|c|}{ Reference Stiffness $(\mathrm{N} / \mathrm{m})$} \\
\cline { 2 - 5 } & 25 & 35 & 45 & 55 \\
\hline $0 T$ & $F<F_{\text {crit }}$ & $F<F_{\text {crit }}$ & $F<F_{\text {crit }}$ & $F<F_{\text {crit }}$ \\
\hline $0.4 T$ & $F<F_{\text {crit }}$ & $F<F_{\text {crit }}$ & $F<F_{\text {crit }}$ & $F<F_{\text {crit }}$ \\
\hline $0.5 T$ & $F<F_{\text {crit }}$ & $F>F_{\text {crit }}$ & $F<F_{\text {crit }}$ & $F<F_{\text {crit }}$ \\
\hline $0.6 T$ & $F>F_{\text {crit }}$ & $F>F_{\text {crit }}$ & $F>F_{\text {crit }}$ & $F>F_{\text {crit }}$ \\
\hline $0.7 T$ & $F<F_{\text {crit }}$ & $F<F_{\text {crit }}$ & $F<F_{\text {crit }}$ & $F<F_{\text {crit }}$ \\
\hline $0.8 T$ & $F<F_{\text {crit }}$ & $F<F_{\text {crit }}$ & $F<F_{\text {crit }}$ & $F<F_{\text {crit }}$ \\
\hline $1.0 T$ & $F<F_{\text {crit }}$ & $F<F_{\text {crit }}$ & $F<F_{\text {crit }}$ & $F<F_{\text {crit }}$ \\
\hline
\end{tabular}

To compare the performances between the three positions, Analysis of Variance (ANOVA) was used to confirm a significant difference, shown in Table 1. In this table, $F$-value is calculated by the ANOVA test to examine whether the means between two conditions are significantly different or not, where the threshold is taken as $F_{\text {crit }(0.05,3,15)}=3.28$. According to Table $1, F>F_{\text {crit }}$ indicates a significant difference is observed for this comparison. Furthermore, Tukey's range test is used to find a pair of difference after the ANOVA test. $q$-value is calculated by the differences between means of two conditions to compare with $q_{\text {crit }(0.05,3,15)}=3.67$. In Table $2, q>q_{\text {crit }}$ shows the significant difference in each pair. Darker-color blocks in both tables show the significant difference in each comparison with $p<0.05$. The ANOVA test reveals a significant difference from 


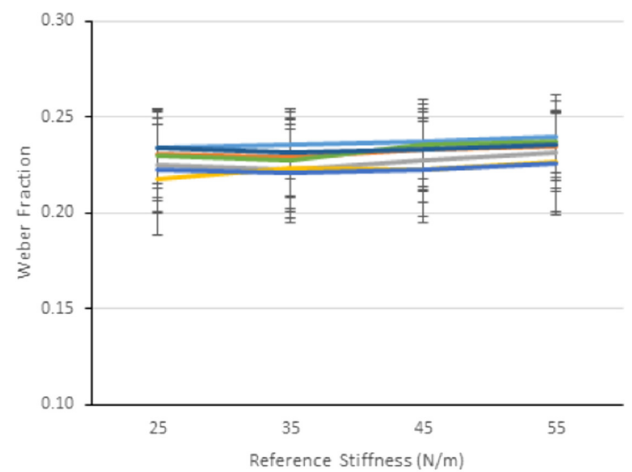

(a)

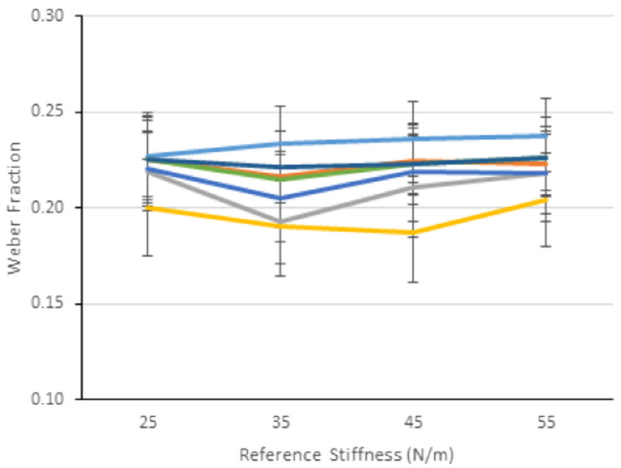

(b)

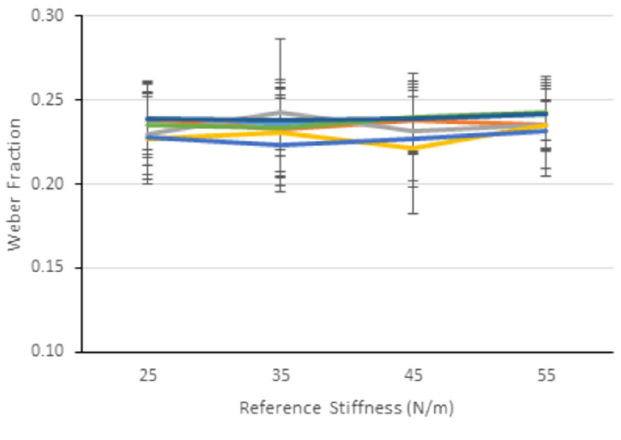

$-0 \mathrm{~T}-0.4 \mathrm{~T}-0.5 \mathrm{~T}-0.6 \mathrm{~T}-0.7 \mathrm{~T}-0.8 \mathrm{~T}-1.0 \mathrm{~T}$

(c)

Fig. 3. User performance in each position of the vibration source (a) on the index finger, (b) between index finger and the thumb, (c) on the thumb. 
Table 2. Results of Tukey's range test in the comparison of the position of vibration source

\begin{tabular}{|c|c|c|c|c|}
\hline \multicolumn{5}{|c|}{ Tukey's Range Test } \\
\hline Vibration Intensity & Reference Stiffness $(\mathrm{N} / \mathrm{m}$ & Pos. 1 - Pos. 2 & Pos.1 - Pos. 3 & Pos.2 - Pos. 3 \\
\hline $0.5 T$ & 35 & $q<q_{\text {crit }}$ & $q<q_{\text {crit }}$ & $q>q_{\text {crit }}$ \\
\hline \multirow{4}{*}{$0.6 T$} & 25 & $q<q_{\text {crit }}$ & $q<q_{\text {crit }}$ & $q>q_{\text {crit }}$ \\
\hline & 35 & \multirow{2}{*}{$\begin{array}{l}q>q_{\text {crit }} \\
q>q_{\text {crit }}\end{array}$} & $q<q_{\text {crit }}$ & $q>q_{\text {crit }}$ \\
\hline & 45 & & $q<q_{\text {crit }}$ & $q>q_{\text {crit }}$ \\
\hline & 55 & $q<q_{\text {crit }}$ & $q<q_{\text {crit }}$ & $q>q_{\text {crit }}$ \\
\hline
\end{tabular}

Pos.1, Pos.2, and Pos.3 are Position 1, Position 2, and Position 3, respectively.

the position when the intensity is at $0.5 T$ in $35 \mathrm{~N} / \mathrm{m}$ and $0.6 T$ in all reference stiffnesses. Then the results of the Tukey's test in Table 2 also show significant differences against Position 2 for Position $1\left(q>q_{\text {crit }}\right)$ when the vibration is at $0.6 T$ in all reference stiffnesses and $0.5 T$ at $35 \mathrm{~N} / \mathrm{m}$. Furthermore, in comparison between Position 1 and Position 2, significant differences $\left(q>q_{\text {crit }}\right)$ are also observed when the intensity is at $0.6 T$ in both 35 , and $45 \mathrm{~N} / \mathrm{m}$.

The present study showed the SR improves the fingertip sensation of both fingers even when the fingers are within the holders. It is hypothesized that when the vibration source is on Position 2, the vibration propagates to both fingers and not only a single finger as the other two conditions. Furthermore, the results indicated the potential of one vibration source at a remote position being able to improve the sensations of the two fingers. In addition, the limitation of this study is that the test subjects are still small in number and there is no variety of gender and age of the participants. Moreover, the subject in other conditions, such as stroke and other disability in haptic sensation, are not examined. Further investigations would be necessary, including the possibility of the improvement of the haptic performance through stochastic resonance.

\section{Conclusion}

We proposed a novel method for emphasizing the stiffness discrimination ability with multiple fingers in VE. The proposed method combined haptic feedback and the effect of SR in order to enhance the haptic performance while carrying out the task. The experimental results show the increase of the performance while applying a subthreshold vibration. Therefore, the proposed method is believed to enhance the sensation of two fingers in stiffness discrimination task with one vibration source when the fingers are inserted in the holders by the effect of SR. In future work, we will investigate the potential of this application and other possibilities in order to enhance haptic performances.

Acknowledgment. This work was supported in part by KAKENHI Grant No. $17 \mathrm{~K} 00270$ and $20 \mathrm{H} 04227$. 


\section{References}

1. Lederman, S.J., Klatzky, R.L.: Sensing and displaying spatially distributed fingertip forces in haptic interfaces for teleoperator and virtual environment systems. Presence Teleoperators Virtual Environ. 8(1), 86-103 (1999)

2. Karol, S., Koh, K., Kwon, H.J., Park, Y.S., Kwon, Y.H., Shim, J.K.: The effect of Frequency of Transcutaneous Electrical Nerve Stimulation (TENS) on maximum multi-finger force production. Korean J. Sport Biomech. 26(1), 93-99 (2016)

3. E, Sens., et al.: Effects of temporary functional deafferentation on the brain, sensation, and behavior of stroke patients. J. Neurosci. 32(34), 11773-11779 (2012)

4. Smith, A.: Effects of caffeine in chewing gum on mood and attention. Hum. Psychopharmacol. Clin. Exp. 24(3), 239-247 (2009)

5. Dettmer, M., Pourmoghaddam, A., Lee, B.C., Layne, C.S.: Effects of aging and tactile stochastic resonance on postural performance and postural control in a sensory conflict task. Somatosens. Motor Res. 32(2), 128-135 (2015)

6. Collins, J.J., Imhoff, T.T., Grigg, P.: Noise-mediated enhancements and decrements in human tactile sensation. Phys. Rev. E 56(1), 923 (1997)

7. Cornsweet, T.N.: The staircase-method in psychophysics. Am. J. Psychol. 75(3), 485-491 (1962)

8. Kurita, Y., Shinohara, M., Ueda, J.: Wearable sensorimotor enhancer for fingertip based on stochastic resonance effect. IEEE Trans. Hum. Mach. Syst. 43(3), 333$337(2013)$

9. Enders, L.R., Hur, P., Johnson, M.J., Seo, N.J.: Remote vibrotactile noise improves light touch sensation in stroke survivors' fingertips via stochastic resonance. J. Neuroengineering. Rehabil. 10(1), 105 (2013)

10. Chamnongthai, K., Endo, T., Nisar, S., Matsuno, F., Fujimoto, K., Kosaka, M.: Fingertip force learning with enhanced haptic sensation using stochastic resonance. In: Proceedings of the IEEE World Haptics Conference, pp. 539-544 (2019)

11. Conti, F., et al.: The CHAI libraries. In: Proceedings of the Eurohaptics, pp. 496$500,(2003)$

12. Johansson, R.S., Landstro, U., Lundstro, R.: Responses of mechanoreceptive afferent units in the glabrous skin of the human hand to sinusoidal skin displacements. Brain Res. 244(1), 17-25 (1982)

13. Box, G.E.P.: A note on the generation of random normal deviates. Ann. Math. Stat. 29, 610-611 (1958)

14. Samani, A., Zubovits, J., Plewes, D.: Elastic moduli of normal and pathological human breast tissues: an inversion-technique-based investigation of 169 samples. Phys. Med. Biol. 52(6), 1565 (2007)

15. Taylor, M., Creelman, C.D.: PEST: efficient estimates on probability functions. J. Acoust. Soc. Am. 41(4A), 782-787 (1967) 
Open Access This chapter is licensed under the terms of the Creative Commons Attribution 4.0 International License (http://creativecommons.org/licenses/by/4.0/), which permits use, sharing, adaptation, distribution and reproduction in any medium or format, as long as you give appropriate credit to the original author(s) and the source, provide a link to the Creative Commons license and indicate if changes were made.

The images or other third party material in this chapter are included in the chapter's Creative Commons license, unless indicated otherwise in a credit line to the material. If material is not included in the chapter's Creative Commons license and your intended use is not permitted by statutory regulation or exceeds the permitted use, you will need to obtain permission directly from the copyright holder. 